\title{
Deviation of nuclear radii from a smooth $A$ dependence for neutron data
}

\author{
F.S. Dietrich ${ }^{1, a}$, J.D. Anderson ${ }^{1}$, R.W. Bauer ${ }^{1}$, M. Girod ${ }^{2}$, D. Gogny ${ }^{1}$, S.M. Grimes ${ }^{3}$, S. Hilaire ${ }^{2}$, and D.P. McNabb ${ }^{1}$ \\ 1 Lawrence Livermore National Laboratory, Livermore, CA 94550, USA \\ 2 CEA/DAM Île-de-France, DPTA/SPN, BP. 12, 91680 Bruyères-le-Châtel, France \\ 3 Ohio University, Athens, OH 45701, USA
}

\begin{abstract}
Experimental values for r.m.s. charge density radii show deviations from a simple $A^{1 / 3}$ dependence of several percent for statically deformed nuclei and around shell closures. These data, for both stable nuclei and nuclei away from the valley of stability, are well described by Hartree-Fock-Bogolyubov calculations carried out at Bruyèresle-Châtel. By using the neutron and proton r.m.s. radii from these same HFB calculations in conjunction with a folding model to produce an optical potential, we can then predict the expected results from neutron scattering experiments. We have made a detailed study of this procedure in the $A=40-70$ mass range. The calculated $14-\mathrm{MeV}$ neutron nonelastic and total cross sections show deviations of several percent from a smooth dependence on $A$. We conclude that using mean field - folding model calculations is useful for estimating neutron cross sections on nuclei both on and away from the valley of stability.
\end{abstract}

\section{Introduction}

The radius of the real part of the optical potential is one of the key parameters in determining cross sections for neutron interactions with nuclei. In global optical potentials the radius is parameterized as a smooth function of $A$, typically $R=r_{0} A^{1 / 3}$. In the microscopic picture underlying the optical potential, the potential is related to the neutron and proton densities of the target nucleus. However, these densities have radii that show significant fluctuations with $A$, due to shell and other structure effects including deformation. This is best illustrated by the r.m.s. charge radii, which have been extensively studied by a variety of electromagnetic probes, for nuclei removed from the valley of stability by several nucleons as well as for stable nuclei. These results have been catalogued in evaluations such as those by Nadjakov et al. [1] and Angeli [2]. It is expected that deviations from a smooth $A$ dependence seen in these data should be mirrored in the optical-potential radius, since at reasonably low energies (below about $50 \mathrm{MeV}$ ) neutron scattering is predominantly sensitive to the protons in the target.

Global optical potentials clearly lack the ability to reproduce local fluctuations in radii. However, individual fits to nuclei are not likely to be satisfactory either, since small variations of the radius can easily be obscured by vaariations in other parameters in a multiparameter fit. To improve this situation, we examine the ability of a particular microscopic treatment of nuclear structure (the Hartree-Fock-Bogolyubov [HFB] theory as implemented at Bruyères-le-Châtel using the D1S interaction $[3,4]$ ) to reproduce the r.m.s. charge radii. Since we find the charge radii are very well reproduced by the calculations, we then use the r.m.s. neutron and proton radii predicted by these same calculations to generate a neutron optical potential, using a folding model based on the JLM optical potential [5-7]. We show the r.m.s. radii of the real potential generated by this procedure for $14-\mathrm{MeV}$ neutrons in the $A=40-70$ mass region, as well as the predicted

${ }^{a}$ Presenting author, e-mail: dietrich2@llnl.gov nonelastic cross sections. The nonelastic cross sections show significant (5-10\%) deviations from a smooth dependence on $A$, which strongly supports the utility of the HFB calculations, in conjunction with a folding model, for determining cross sections for nuclei off the valley of stability.

\section{Behavior of the r.m.s. charge radii}

The measured r.m.s. charge radii divided by $A^{1 / 3}$ for stable nuclei from compilation [1] are shown in the upper panel of figure 1 as a function of $A^{1 / 3}$. These show $2 \%$ deviations from a smooth behavior represented by the reference line. Major deviations occur in the regions where static deformations occur, around $A^{1 / 3}=5.5$ and $A^{1 / 3}>6$. We apply a correction for deformation as given by Bohr and Mottelson [8] by dividing by $\left[1+(5 / 4 \pi) \beta^{2}\right]^{1 / 2}$, using tabulated values for the deformation parameters $\beta$. In the lower portion of figure 1 we see that the deviations have been reduced by a factor of approximately 4 . The reference line is the same in both parts of the figure.

The charge radii in the HFB calculations are taken at a deformation that minimizes the energy of the nucleus, and thus may be expected to include effects of deformation in a natural way, as well as those from single-particle shell structure. Figure 2 shows the ratio of the measured to calculated r.m.s. charge radii for stable nuclei from compilation [1] (with a number of points having large uncertainties removed). Deviations from unity are largely below $0.5 \%$. We have also compared the HFB calculations with an extensive body of isotope-shift measurements of the charge radii of isotopic sequences tabulated in [1] and [2]. The calculations reproduce these data, primarily for nuclei off the valley of stability, very well. An example, for the barium isotopes, is shown in figure 3, where the HFB calculations of the r.m.s. charge radii divided by $A^{1 / 3}$ (solid line) are compared with the measurements (squares). The calculations account very well for the shell closure for 82 neutrons at $A=138$. 


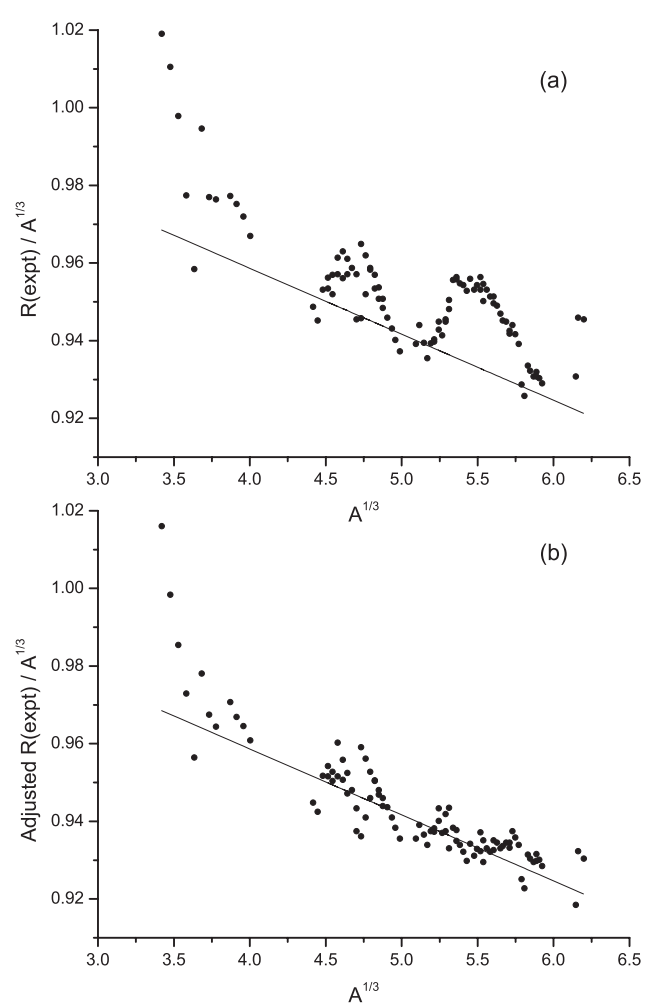

Fig. 1. (a) Measured r.m.s. charge radii divided by $A^{1 / 3}$ as a function of $A^{1 / 3}$ from compilation [1]. (b) Same, but with a correction for nuclear deformation; see text. The solid reference lines are identical in both parts of the figure.

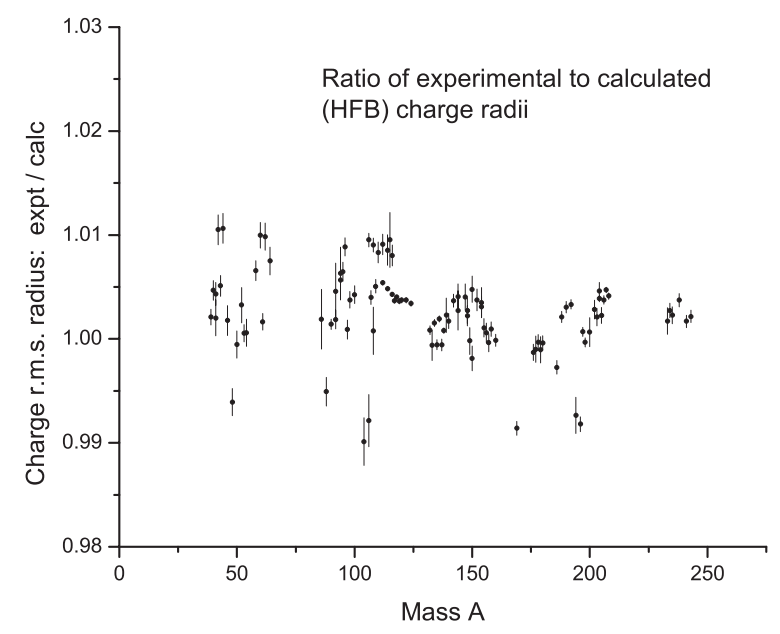

Fig. 2. Measured r.m.s. charge radii from [1] divided by the charge radii from the Hartree-Fock-Bogolyubov calculations.

The effects of shell closure in the mass region $A=40-70$ are shown in figure 4 . The upper portion of the figure shows experimental values for the r.m.s. charge radii divided by $A^{1 / 3}$. For fixed $A$, there are large radius differences $(\sim 1.5-$ $4 \%$ ) among the various elements for $A=48,50$, and 64 corresponding to the shell closures at $N=28$ and $Z=28$. In the lower part of the figure, the experimental values of the radii are divided by the results of the HFB calculations. We

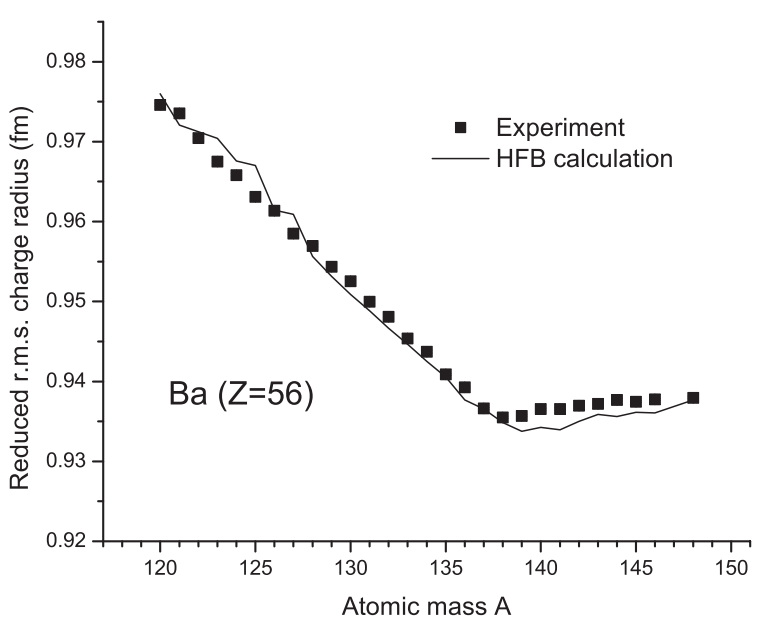

Fig. 3. Hartree-Fock-Bogolyubov calculations of the r.m.s. charge radii for barium isotopes divided by $A^{1 / 3}$ (solid line) are compared with the results of isotope-shift measurements (squares).

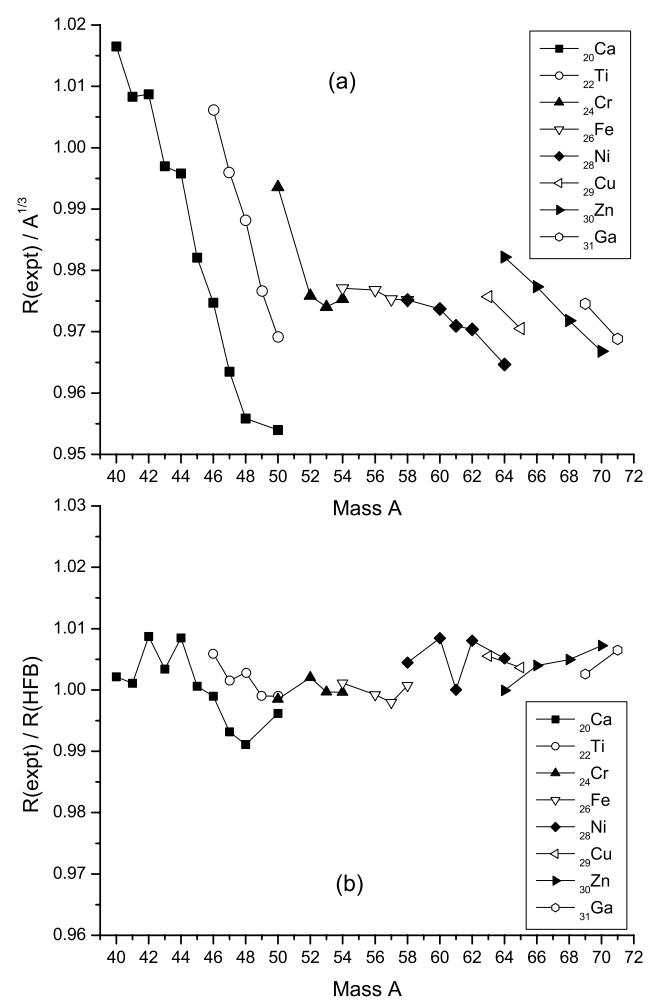

Fig. 4. (a) Experimental values for the r.m.s. charge radii divided by $A^{1 / 3}$ in the mass region $A=40-70$. (b) Same, divided by the results of the HFB calculations.

note the ability of the HFB calculations to reproduce the large fluctuations shown in the upper part of the figure.

\section{Implications for neutron-induced reactions}

In the previous section we have shown that the HFB calculations account for the observed r.m.s. radii of charge distributions at the level of $0.5 \%$ or better for $A \geq 40$. 


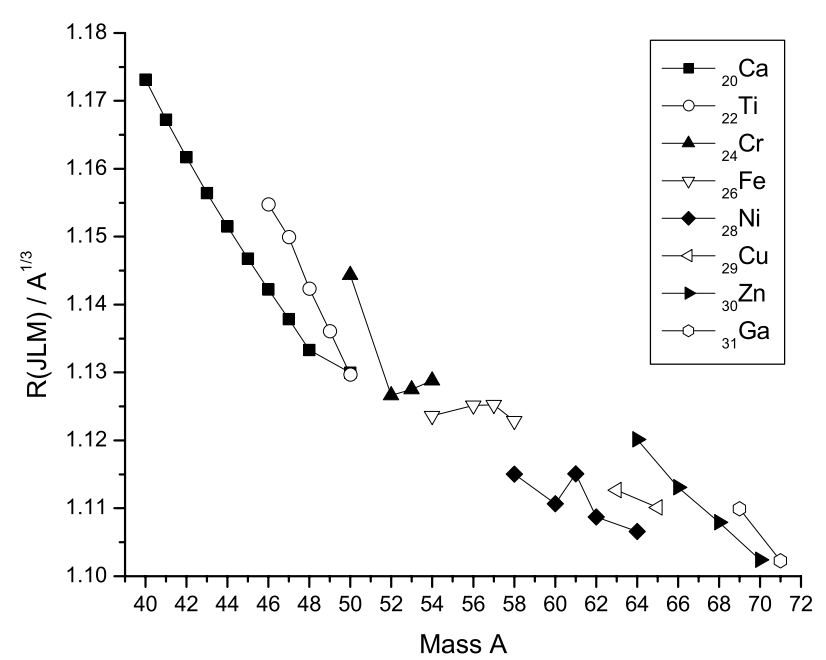

Fig. 5. R.m.s. radii divided by $A^{1 / 3}$ of the real part of the central optical potential for $14-\mathrm{MeV}$ neutrons in the $A=40-70$ region. The potential was calculated by a folding model with densities determined by the HFB calculations as described in the text.

This result strongly suggests that the r.m.s. neutron and proton radii given by these calculations should be reliable. We use these radii to determine the radius parameters of Woods-Saxon functions representing the neutron and proton density distributions, assuming a constant diffuseness parameter of $0.54 \mathrm{fm}$. These densities are then used in a foldingmodel calculation of the optical potential for $14-\mathrm{MeV}$ neutrons using an effective interaction based on the JLM nuclear-matter optical potential [5-7]. The details of the calculations are identical to those used to calculate total cross sections in the paper of Abfalterer et al. [9]. We have carried out these calculations in the $A=40-70$ region that showed strong shell effects in the charge radii. In principle the calculations could be carried out with the full neutron and proton density distributions given by the HFB calculations, as has been done in numerous earlier calculations (see, for a recent example, ref. [10]). The current procedure is a useful simplification in the present context, since we are interested in the effects of the overall size of the neutron and proton distributions, rather than in fine details of the radial distributions.

The r.m.s. radii (divided by $A^{1 / 3}$ ) of the real part of the central optical potential calculated by the above procedure are shown in figure 5 . The general behavior of these radii is similar to that of the r.m.s. charge radii shown in the upper part of figure 4 . This is not surprising, since at $14 \mathrm{MeV}$ the interaction of the projectile neutron with the target protons is much stronger than with the target neutrons.

The nonelastic (reaction) cross sections calculated from these potentials are shown in figure 6. In this figure the cross sections are divided by a factor representing the nuclear area, $\pi\left(r_{0} A^{1 / 3}+1 / k\right)^{2}$, where $r_{0}=1.4 \mathrm{fm}$ and $k$ is the wave number. The nonelastic cross sections also reflect the behavior of the charge radii. The predicted cross sections (divided by the area factor) differ very strongly from the constant value that would be expected in the absence of shell and isospin effects in the optical potential. Similar behavior, not shown here, is seen in the predicted total cross sections. These effects, which are

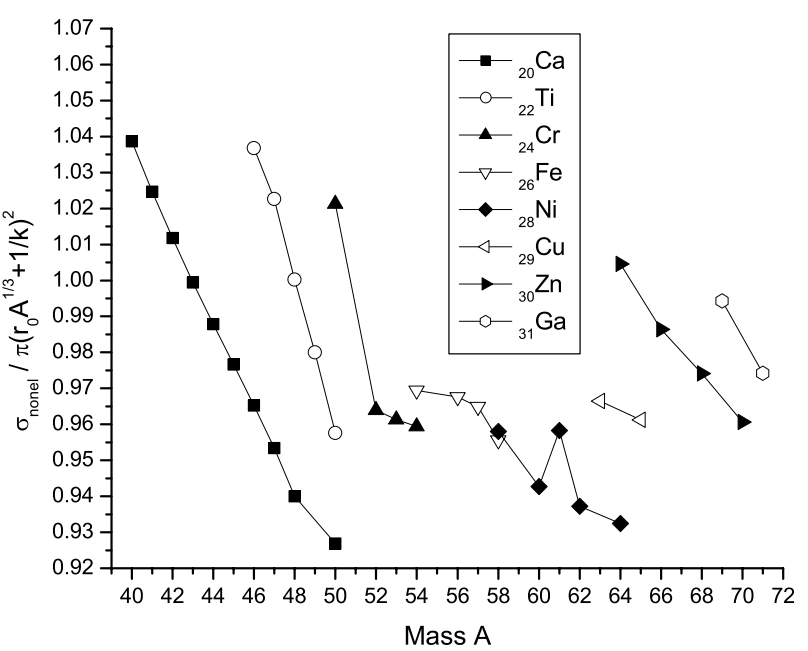

Fig. 6. Nonelastic cross sections for $14-\mathrm{MeV}$ neutrons in the $A=40$ 70 mass region determined by a folding-model potential as described in the text. The cross sections are divided by a factor representing the area of the nucleus.

as large as $5-10 \%$ in the nonelastic cross sections, are large enough to be observable in careful experiments, at least for stable nuclei.

\section{Summary and conclusions}

We have studied the systematics of measured r.m.s. charge radii in comparison with the results of microscopic structure (Hartree-Fock-Bogolyubov) calculations. Using a folding model to determine an optical potential, we have used these results to predict the size of shell and isospin effects in neutron scattering observables.

For charge radii:

- R.m.s. charge radii show $\sim 2 \%$ or greater deviations from $A^{1 / 3}$ near closed shells and in regions of large static deformation.

- These deviations are well described by the HFB calculations (and probably by other mean field techniques as well, if carried out in sufficient detail).

For neutron reaction observables:

- R.m.s. radii of the $14-\mathrm{MeV}$ neutron potentials calculated with the folding model in the $A=40-70$ range show similar behavior to the charge radii.

- The corresponding nonelastic cross sections at $14 \mathrm{MeV}$ show deviations at the $5-10 \%$ level from proportionality to $A^{2 / 3}$

We conclude that mean-field techniques such as the HFB calculations used here are accurate enough to serve as a basis for microscopic treatments of neutron scattering. The shell and deformation effects contained in such calculations are large enough to yield significant deviations from smooth behavior with $A$ in scattering observables.

This work was performed in part under the auspices of the US Department of Energy (DOE) by the University of California, Lawrence Livermore National Laboratory (LLNL) under contract No. W-7405Eng-48. 


\section{References}

1. E.G. Nadjakov, K.P. Marinova, Y.P. Gangrsky, At. Data Nucl. Data Tables 56, 133 (1994).

2. I. Angeli, At. Data Nucl. Data Tables 87, 185 (2004).

3. J. Dechargé, D. Gogny, Phys. Rev. C 21, 1568 (1980).

4. J.F. Berger, M. Girod, D. Gogny, Comp. Phys. Comm. 63, 365 (1991).

5. J.P. Jeukenne, A. Lejeune, C. Mahaux, Phys. Rev. C 10, 1391 (1974).
6. J.P. Jeukenne, A. Lejeune, C. Mahaux, Phys. Rev. C 15, 10 (1977).

7. J.P. Jeukenne, A. Lejeune, C. Mahaux, Phys. Rev. C 16, 80 (1977).

8. A. Bohr, B. Mottelson, Nuclear Structure, Vol. 1 (Benjamin, New York, 1969).

9. W.P. Abfalterer, F.B. Bateman, F.S. Dietrich, R.W. Finlay, R.C. Haight, G.L. Morgan, Phys. Rev. C 63, 044608 (2001).

10. F.S. Dietrich, J.D. Anderson, R.W. Bauer, S.M. Grimes, R.W. Finlay, W.P. Abfalterer, F.B. Bateman, R.C. Haight, G.L. Morgan, E. Bauge et al., Phys. Rev. C 67, 044606 (2003). 